\title{
BCG Vaccination Protection Against COVID-19: Correspondence
}

Received: 6 October 2020 / Accepted: 18 November 2020 / Published online: 20 January 2021

(C) Dr. K C Chaudhuri Foundation 2021

To the Editor: We read the article "BCG Vaccination Policy and Protection Against COVID-19" published by Meena et al. [1] (The Indian Journal of Pediatrics, https://doi.org/10.1007/ s12098-020-03371-3) with great interest. We congratulate the authors for analyzing the correlation between $B C G$ vaccination and COVID-19 cases and mortality. The authors were unable to find any significant correlation between COVID-19 cases and deaths per million population with BCG vaccination coverage rates. However, correlation does not guarantee association or prove anything until unless compared with control group. Authors did not compare the mortality rates and COVID-19 cases with countries without any BCG vaccination policy. Authors mentioned that they adjusted for confounding variables. However, authors were unable to mention the method used for adjustment of covariates and $p$-values after adjustment. Kindly share the test used for adjustment of confounding factors and how did they conclude no association with $B C G$ vaccination and COVID-19 mortality. Escobar et al. [2] found the negative association between BCG vaccination and COVID-19 mortality in socio-economic similar European countries (BCG vaccination versus no BCG vaccination) and port of entry of US (No BCG recommendation) and South American countries (BCG vaccination recommendation). Author's conclusion about spurious association of studies like Klinger et al. [3] despite adjustment of covariates and having control group may be misleading to readers.

We appreciate the input of authors in conducting the analysis. However, with no control group and not specifying the method of adjustment of covariates, we are of the opinion that there are certain issues pertaining to assessment of evidence, which needs to be clarified. We also wish to clarify that by no means this is an attempt to disregard the hard work of the authors.

\footnotetext{
Surjit Singh $^{1}$, Daisy Khera ${ }^{2}$, and Ankita Chugh ${ }^{3}$

${ }^{1}$ Department of Pharmacology, ${ }^{2}$ Department of Pediatrics,

${ }^{3}$ Department of Dentistry, All India Institute of Medical Sciences, Jodhpur, Rajasthan, India. Email: daisykhera78@gmail.com
}

\section{References}

1. Meena J, Yadav A, Kumar J. BCG vaccination policy and protection against COVID-19. Indian J Pediatr 2020;87(9):749.

2. Escobar LE, Molina-Cruz A, Barillas-Mury C. BCG vaccine protection from severe coronavirus disease 2019 (COVID-19). Proc Natl Acad Sci U S A 2020;117(30):17720-6.

3. Klinger D, Blass I, Rappoport N, Linial M. Significantly improved COVID-19 outcomes in countries with higher BCG vaccination coverage: a multivariable analysis. Vaccines (Basel). 2020;8(3):378.

\section{Authors' Reply}

To the Editor: We appreciate the interest of the authors in our article and we intend to utilize this opportunity to respond to their queries [1].

We disagree with the readers' comments "correlation doesn't guarantee association or prove anything until unless compared with control group". We did not equate correlation and association (In fact, we did not even use the word "association"). Moreover, corelation is a specific type of association, but not causation [2]. Also, to stress that neither association nor correlation of BCG and COVID-19 guarantee causation [3]. Also, we would like to emphasize here that the correlation does not need a control group. For two continuous outcomes (for which there are no valid predefined categories), correlation is an important statistical measure to ascertain the relationship. Furthermore, most of the studies on this aspect used correlation co-efficients only (including Klinger et al. study mentioned by readers) [4].

We did not compare the countries without any BCGvaccination policy in our analysis as only 13 countries did not have a universal BCG vaccination policy at any timepoint from 1980 to 2018 (as mentioned in the manuscript). Among these 13 countries, many did not have a significant number of COVID-19 cases at that time. In our paper [1], we also mentioned the reasons for not categorizing the countries based on recent vaccination status.

The readers' comments that we "were unable to mention" the method used for adjustment of covariates and 
$p$-values after adjustment is distasteful. We used regression analysis, a well-known and basic statistical test. Being a standard biostatistical method for such analysis, we did not report separately considering constrain of word limit.

Furthermore, it is unfortunate that the readers have interpreted our conclusions incorrectly. We chose our words very carefully and mentioned that there is no meaningful relationship (correlation co-efficient is a measure of relationship). Therefore, the reader's comment "we concluded that there is no association with BCG vaccination and COVID19 mortality" is incorrect.

Also, the readers mentioned that "the author's conclusion about the spurious association of studies like Klinger et al despite adjustment of covariates and having control group may be misleading to readers". The fact is that we did not even quote this study in our manuscript at all as this study has a cohort of countries with no routine BCG vaccination policy only in the last $15 \mathrm{y}$. We mentioned that classifying countries based upon recent vaccination status and just the mere presence of BCG vaccination policy despite very low coverage may lead to spurious associations. A large single country study as well as an epidemiological analysis of homogenous population also did not find any such association $[4,5]$.

At last, we again disagree with the distasteful comment "however, with no control group and not specifying the method of adjustment of co-variates, we are of the opinion that there are certain issues pertaining to the assessment of evidence, which needs to be clarified" and sincerely hope that all the queries are clarified.

Jitendra Meena, Jogender Kumar, and Arushi Yadav

Neonatology Division, Department of Pediatrics, Post Graduate Institute of Medical Education and Research, Chandigarh, India. E-mail: jogendrayadv@gmail.com

\section{References}

1. Meena J, Yadav A, Kumar J. BCG vaccination policy and protection against COVID-19. Indian J Pediatr. 2020;87(9):749.

2. Altman N, Krzywinski M. Association, correlation and causation. Nat Methods. 2015;12:899-900.

3. Kumar J, Meena J. Demystifying BCG vaccine and COVID-19 relationship. Indian Pediatr. 2020;57(6):588-9.

4. Lindestam Arlehamn CS, Sette A, Peters B. Lack of evidence for BCG vaccine protection from severe COVID-19. Proc Natl Acad Sci U S A. 2020;117:25203-4.

5. Hamiel U, Kozer E, Youngster I. SARS-CoV-2 rates in BCG-vaccinated and unvaccinated young adults. JAMA. 2020;323(22):2340-1.

Publisher's Note Springer Nature remains neutral with regard to jurisdictional claims in published maps and institutional affiliations. 